Conclusion The laparoscopic approach to distal pancreatectomy results in significantly lower blood loss, and shorter HDU and hospital stay compared with open surgery. The postoperative morbidity and $\mathrm{R} 0$ resection margin rates were comparably similar.

Competing interests None declared.

\section{PM0-100 OUTCOME OF MINIMALLY INVASIVE PANCREATIC NECROSECTOMY WITHOUT IRRIGATION}

doi:10.1136/gutjnl-2012-302514b.100

D J Malde, ${ }^{*}$ S S Raza, N Khan, A Aldouri, K Menon, A M Smith. The HPB Unit, St James University Hospital, Leeds, UK

Introduction Necrotizing Pancreatitis with secondary infection of the pancreatic tissue is associated with significant morbidity and mortality. Current evidence suggests that a minimally invasive retroperitoneal necrosectomy (MIRP) is feasible, well tolerated and beneficial for the patient when compared with open surgery.

Methods A total of 16 patients who underwent MIRP from September 2007 to April 2011 were included in the study. Current minimal access techniques all recommend routine irrigation but we aim to show that comparable results can be achieved without irrigation.

Results The mean age was 52.5 years with 13 patients transferred from other centres. The aetiology was gallstones (13), alcohol (1), idiopathic (1) and hyperlipidaemia (1). The average time before 1st necrosectomy was 50.2 days. The mean number of procedures was 3.3 (range $1-7$ ) with one patient requiring an open procedure. One patient required post-necrosectomy ICU admission. 13 patients had nasojejunal feed and four patients started with parenteral feed which was later converted to nasojejunal. Five patients developed a pancreatic fistula, three patients developed colonic fistula and two patients died. Mean inpatient stay was 82.6 days (range 31-182).

Conclusion This series suggests that doing MIRP without irrigation has results comparable to other centres carrying out routine irrigation.

Competing interests None declared.

\section{PM0-101 ENTERAL NUTRITION IN ACUTE PANCREATITIS: NASOGASTRIC OR NASOJEJUNAL?}

\section{doi:10.1136/gutjnl-2012-302514b.101}

D J Malde, ${ }^{*}$ A Suppiah, T Arab, K Menon, A M Smith. The HPB Unit, St James University Hospital, Leeds, UK

Introduction Enteral feeding is beneficial in patients with severe acute pancreatitis. A published series suggests $100 \%$ can be fed via the nasogastic (NG) route.

Methods 146 consecutive patients (January-December 2010) admitted with acute pancreatitis (AP) were reviewed to assess the safety and tolerance of $\mathrm{NG}$ feed. In all severe AP patients nutrition was initially delivered via an NG tube, if they were not absorbing a nasojejunal (NJ) tube was inserted.

Results 29 patients were identified as having poor outcome. 127 (87\%) patients were able to commence oral intake within $72 \mathrm{~h}$ of admission. $19(13 \%)$ patients required additional enteral or parenteral nutritional support. 16 patients were commenced on NG feed but two patients needed conversion to $\mathrm{NJ}$ feed. Three patients were directly commenced on NJ feed but one needed conversion to parenteral feed. Only one patient had been commenced on parenteral feed prior to transfer. Need for nutritional support was a significant indicator of poor outcome; morbidity $13 / 19$ vs $12 / 127$ $(p<0.0001)$ and mortality $6 / 19$ vs $1 / 127(p<0.0001)$.
Conclusion Nasogastric feeding is well tolerated in the majority (73.7\%) of patients with severe AP. NG feeding should be first line, but if failing a rapid change to the NJ route instituted.

Competing interests None declared.

\section{PMO-102 MEMBRANOUS EXPRESSION OF SULFATASE-2 IS ASSOCIATED WITH A POORER PROGNOSIS IN PATIENTS FOLLOWING PANCREATIC CANCER RESECTION}

doi:10.1136/gutjnl-2012-302514b.102

${ }^{1} \mathrm{D}$ Televantou, ${ }^{*}{ }^{1,2} \mathrm{~K}$ Y D Hui, ${ }^{3} \mathrm{R}$ Lochan, ${ }^{3} \mathrm{R}$ Charnley, ${ }^{4} \mathrm{~A} \mathrm{D}$ Burt, ${ }^{1,5} \mathrm{H}$ L Reeves. ${ }^{1}$ Northern Institute for Cancer Research, Newcastle University, Newcastle upon Tyne, UK; ${ }^{2}$ Department of HPB and Transplant Surgery, Freeman Hospital, Newcastle upon Tyne, UK; ${ }^{3}$ Department of Surgery, Freeman Hospital, Newcastle upon Tyne, UK; ${ }^{4}$ Institute of Cellular Medicine, Newcastle University, Newcastle upon Tyne, UK; ${ }^{5}$ Department of Hepatology, Freeman Hospital, Newcastle Upon Tyne, UK

Introduction Pancreatic adenocarcinomas are resistant to medical therapies and associated with a poor prognosis. Sulfatase 2 (SULF2) is one of two extracellular heparan sulphate 6-O-endosulfatases that modulate ligand activated FGF and Wnt signalling. SULF2 expression is dramatically upregulated at mRNA levels in pancreatic cancers (NCBI GEO). We have investigated SULF2 protein expression in pancreatic adenocarcinomas, in association with clinicopathological parameters.

Methods Immunohistochemistry for SULF2 was performed on archived FFPE (Formalin-Fixed paraffin Embedded) blocks from 21 resected primary pancreatic adenocarcinomas, most of which were histologically defined as ductal (19/21, 90.5\%). Membranous and cytoplasmic expression of SULF2 in tumour and stromal cells were separately assessed. Additionally, immunostaining for $\boldsymbol{\alpha}$-Smooth Muscle Actin (a-SMA) was performed for further cell characterisation. Results SULF2 was expressed in tumour cells in the majority of the tumours $(18 / 21,86 \%)$. This expression was either cytoplasmic (13/ $21,61.9 \%)$, membranous $(12 / 21,57.1 \%)$ or both $(17 / 21,80.9 \%)$. Membranous positivity was found almost exclusively in tumours with low differentiated areas $(11 / 12, p=0.007)$. Membranous overexpression was also associated with shorter patient survival $(p=0.011)$. Spindle-shaped cells of desmoplastic tumour stroma showed strong cytoplasmic positivity in all tumours studied $(21 / 21$, $100 \%$ ). These cells were also positive for a-SMA, a marker of activated pancreatic stellate cells. Non-neoplastic pancreas showed only focal positivity for SULF2; this involved mainly endothelial, and scattered epithelial cells of exocrine pancreas.

Conclusion SULF2 over- expression is common in pancreatic adenocarcinomas, in both the ductal cancer cells as well as the desmoplastic tumour stroma. Tumour cell membranous localisation and over expression is associated with a more aggressive tumour behaviour and poorer patient survival. SULF2 is a novel candidate biomarker in patients for pancreatic cancer, identifying those with a poorer prognosis, as well as those who may benefit from therapies inhibiting SULF2.

Competing interests None declared.

\section{PMO-103 PROGNOSTIC VALUE OF POST OPERATIVE CA19-9 IN PATIENTS UNDERGOING PANCREATICODUODENECTOMY FOR PANCREATIC ADENOCARCINOMA}

doi:10.1136/gutjnl-2012-302514b.103

D J Malde, E Jeans, ${ }^{*}$ N De Liguori, R Deshpande, B J Ammori, D J Sherlock, D O'Reilly. The HPB Unit, North Manchester General Hospital, Manchester, UK

Introduction Pancreatic Adenocarcinoma accounts for over $90 \%$ of Pancreatic malignancy with overall survival being $<5 \%$ at 5 years. CA $19-9$ is a commonly used tumour marker with levels in excess of $200 \mathrm{U} / \mathrm{ml}$ being $90 \%$ sensitive for pancreatic malignancy. Pre- 